\title{
Experiencias de los estudiantes de estomatologia en el control de la conducta de niños. Analisis cualitativo
}

\section{Experiences of stomatology students in the control of child behavior. Qualitative analysis}

\author{
Valenzuela Muñoz A*, Valenzuela Ramos $M R^{* *}$, Valenzuela Ramos $R^{* * *}$
}

\section{RESUMEN}

Introducción. Uno de los factores que influyen en la atención de los pacientes pediátricos, es la susceptibilidad de los niños a desarrollar con facilidad miedos y temores ante una situación desconocida y sobre todo cuando se trata de la práctica odontológica. Objetivo del estudio es conocer las experiencias de los estudiantes de estomatología en relación al control de la conducta del paciente pediátrico.

Metodología. Para ello fue realizado un análisis cualitativo empleado un enfoque fenomenológico de tipo trascendental. Para lo cual se realizó la entrevista a todos los estudiantes que cumplieron los criterios de selección hasta obtener la saturación de la información. El método de recolección de datos fue la entrevista recomendado por Marshall C 1, y la entrevista siguiendo el enfoque de Seidman I 2 que recomienda realizar tres entrevistas por separado a los participantes.

Resultados. La información obtenida a través de entrevistas y videos fue analizada a través del programa informático Atlas.Ti. Donde se obtiene como resultados la falta de confianza de los estudiantes al momento de atender pacientes pediátricos. Y como se va adquiriendo esta confianza al momento de realizar sus prácticas pre profesionales orientadas por el docente.

Conclusiones. Se concluye que el estudiante no adquiere las habilidades suficientes para lograr el control de la conducta del niño al momento de su atención odontológica, a pesar de tener los conocimientos teóricos. Pero ello se va supliendo siempre y cuando el estudiante toma conciencia y pone en práctica lo aprendido en la teoría.

PALABRAS CLAVE: Estudiantes, Control de la conducta, Niños, Análisis Cualitativo (DeCs).

\section{ABSTRACT}

Introduction. One of the factors that influence the care of pediatric patients is the susceptibility of children to easily develop fears and fears in an unknown situation and especially when it comes to dental practice. Objective of the study is to know the experiences of stomatology students in relation to the control of the behavior of the pediatric patient.

Methodology. For this, a qualitative analysis was carried out in a transcendental phenomenological approach. Why the interview was conducted to all students who met the selection criteria to obtain the saturation of the information. The method of data collection was approved by Marshall C 1, and the interview following the approach of Seidman I 2 that recommends conducting three separate interviews to participants. 
Results. The information obtained through interviews and videos was analyzed through the Atlas.Ti computer program. Where the lack of confidence of the students at the time of attending pediatric patients is obtained as results. And how this confidence is acquired at the moment of carrying out their pre-professional practices guided by the teacher.

Conclusions. It is concluded that the student cannot acquire sufficient skills to achieve control of the child's behavior at the time of dental care, despite having the theoretical knowledge. But this can be provided as long as the student becomes aware and puts into practice what they have learned in theory.

KEY WORDS: Students, Behavior control, Child, Qualitative Analysis.

Fecha de recepción: 21 de enerol de 2019.

Fecha de aceptación: 2 de febrero de 2019.

Valenzuela Muñoz A, Valenzuela Ramos MR, Valenzuela Ramos R. Experiencias de los estudiantes de estomatologia en el control de la conducta de niños. Analisis cualitativo. 2019; 35, (1): 11-17

\section{INTRODUCCIÓN}

“.......los niños son dificiles de atender, su comportamiento cambia, la clase anterior estuve atendiendo a uno de 6 años, yo fui a su casa, para contarle que le iba a curar sus dientecitos, y le dije que si se portaba bien le daría un regalo después de atenderlo, claro le dije delante de su mamá, su mamá también le dijo que le compraría su juguete favorito y el niño dijo que sí que se dejaría atender.........Llego el día de la atención llego el niño Jair con su mamá, pero el niño no quería separarse de su mamá entonces le dije que se suba a sillón , miro a su mamá y ella le dijo que sí que se sentara. Yo tenía todo listo me puse a curarle su diente, pero de repente dijo que ya no quería nada y se paró y se fue corriendo, yo no le hice nada doctora, no le hice daño, hasta tenía su regalo, pero no quería nada. La mamá lo trajo otra vez se sentó y a duras penas termine de curarle. Por eso digo que nunca se sabe cómo va a reaccionar, en cambio un adulto es diferente le explicas y le entiendes, pero un niño no.............."

La persona del testimonio es un estudiante José de estomatología de 26 años de edad que se encuentra en los últimos años de terminar su profesión. En su relato señala lo difícil que es para el manejar la conducta del niño y una de los motivos que el señala es que "el comportamiento del niño cambia y que no se sabe cómo va reaccionar". Así como el, la mayoría de los estudiantes viven experiencias similares cuando atienden a sus pacientes niños por primera vez. Conocen la teoría sobre el control de la conducta del niño para abordarlos en la clínica universitaria estomatológica, pero se les hace difícil llevarlo a práctica, por lo que a veces experimentan una sensación de frustración y por veces abandono del curso. Es común escuchar "mi paciente no se deja atender, llora". Así mismo, observan a sus docentes quienes consiguen atender al paciente aplicando diferentes técnicas, pero cuando ellos lo intentan no pueden realizarlo y acaban por abandonar la asignatura o escoger pacientes definitivamente positivos.

En este sentido, el objetivo del estudio es conocer las experiencias de los estudiantes de estomatología en relación al control de la conducta del paciente pediátrico en las clínicas de estudiantes de dos universidades. El temor de los estudiantes cuando se trata de atender a un paciente pediátrico se debe a diferentes factores.

Uno de los factores que influyen en la atención de los pacientes pediátricos, es la susceptibilidad de los niños a desarrollar con facilidad miedos y temores ante una situación desconocida ${ }^{3}$. El origen de estos miedos frente al tratamiento odontológico probablemente se deba a factores como el estado emocional general del niño, miedos adquiridos (transmitidos por los padres o algunos familiares), experiencias previas negativas y/o dolorosas y la personalidad del niño ${ }^{4}$. Otros factores relacionados con la situación mencionada son la edad, el sexo, la frecuencia y número de visitas al dentista ${ }^{5}$. Todos estos factores pueden influir en la conducta del niño provo- 
cando la aparición de un comportamiento negativo lo que tornará el tratamiento odontológico difícil o imposible de realizar. Otro de los factores que influyen no solo en estudiantes sino también en algunos dentistas no especializados, es que en la mayoría se enfocan en el tratamiento dental, dejando muchas veces de lado la empatía y para lograrlo el profesional debe familiarizarse con el paciente y esto se consigue poniendo en práctica las técnicas de control de la conducta.

El control de la conducta del paciente pediátrico se fundamenta en el conductismo, el cual, plantea que el comportamiento de la persona es modificable si se alteran las circunstancias ambientales que la rodean ${ }^{6}$. En odontología, las técnicas del control de la conducta intentan disminuir el miedo y la ansiedad del niño, con estas técnicas el dentista gana la confianza del niño y logra una buena comunicación, evitando conductas desagradables e improductivas, consiguiendo la colaboración del niño para realizar un tratamiento dental exitoso ${ }^{7,8}$.

La Academia Americana de Odontopediatría ${ }^{9}$ clasifica las técnicas de control de la conducta en básicas, avanzadas y técnicas de modificación de la conducta. Algunos encaminados a mantener la comunicación y otros extinguir conductas inapropiadas. Dentro de las técnicas de control de conducta básica están la comunicación y guía comunicativo, el comportamiento del odontólogo, la accesibilidad del paciente, la presencia/ausencia de los padres, decir - mostrar - hacer, control de voz, comunicación no verbal, el reforzamiento positivo, la distracción, óxido nitroso/inhalación de oxígeno. En las técnicas de control de conducta avanzadas se encuentra la mano sobre boca, restricción física, sedación, anestesia general. Por último, en la técnica de modificación de la conducta están el refuerzo, desensibilización y la imitación. Varios estudios han reportado que las técnicas de imitación y desensibilización son útiles cuando se intenta familiarizar al niño en el ambiente odontológico, logrando el progreso en la conducta del niño en el ámbito dental, facilitando un comportamiento deseable en niños que no han tenido ningún tipo de experiencia dental.

Por otro lado, se toma en cuenta que las experiencias de los estudiantes son singulares, diferentes por lo que no se puede generalizar ${ }^{10}$. Así mismo, se trabaja con sujetos por lo que se requiere tener sensibilidad, empatía y capacidad de escucha ${ }^{10}$. Las experiencias se obtienen a través de sus narraciones, por lo que el investigador debe emplear dispositivos adecuados, de acuerdo a las circunstancias y con los propios sujetos pudiendo ser las entrevistas, los relatos, las autobiografías, la observación participante, la videograbación, entre otras herramientas de investigación de corte cualitativo ${ }^{11}$. Las experiencias de los estudiantes no se encuentran construidas, esto se construirá a través del dialogo y la narración donde el sujeto re-elaborará sus experiencias, expresando emociones y haciendo conexiones de sentido ${ }^{10}$.

En la clínica estomatológica, los estudiantes ponen en práctica los conocimientos adquiridos durante sus estudios pre-clínicos, estas experiencias pueden ser determinantes en su futuro desempeño profesional. La falta de criterio para seleccionar la técnica de control de la conducta del niño adecuada hace el tratamiento difícil o imposible de realizar, para evitar estas dificultades es necesario que el estudiante conozca las técnicas y empatice con el niño. Para la construcción de la experiencia es necesario conocer tres dimensiones centrales: el cuerpo, el tiempo y el espacio. Los estudiantes no son sujetos abstractos, ya que, el cuerpo se interpone sufre y lleva la marca de la experiencia. En relación al tiempo, las experiencias pasadas pueden sufrir una transformación, lo cual dará lugar a una nueva experiencia que dará pie a formular experiencias futuras. Las experiencias no siempre tienen la misma forma que cuando las conocimos, estas pueden estar en constante transformación.

\section{METODOLOGÍA}

En el presente estudio se ha empleado un enfoque fenomenológico de tipo trascendental, el cual aborda la descripción de las experiencias de los participantes más que la interpretación de estas ${ }^{12}$. La pregunta que guio el presente trabajo fue ¿Cómo te sientes atender a pacientes pediátricos? Para responder a la pregunta se entrevistó a estudiantes de dos universidades de la Región Lambayeque, quienes fueron incluidos al estudio por haber aceptado voluntariamente formar parte del estudio, residir durante las prácticas pre-profesionales en la Región Lambayeque, haber firmado el consentimiento informado y que por primera vez atendían a niños en la Clínica Estomatológica. Se realizó la entrevista a todos los estudiantes que cumplieron los criterios de selección hasta obtener la saturación de la información ${ }^{13}$. De los cuales 18 fueron mujeres y 12 
varones, cuatro estaban casado y diez tenían hijos pequeños. Los estudiantes eran de distintas regiones del Perú. Las características demográficas se definieron por conveniencia con el fin de lograr una comprensión y descripción de sus experiencias.

El método de recolección de datos fue la entrevista recomendado por Marshall $C^{1}$, quienes señalan que esta es recomendable para un estudio de tipo fenomenológico, a su vez, basamos la entrevista siguiendo el enfoque de Seidman $\mathrm{I}^{2}$ que recomienda realizar tres entrevistas por separado a los participantes. Se realizó tres entrevistas a cada uno de los participantes, la primera cuando el estudiante inicio el ciclo académico y nunca había atendido a un paciente niño, la segunda a la mitad del ciclo académico y la tercera al finalizar el ciclo académico.

Se analizó los datos de la Investigación utilizando el programa ATLAS.ti, versión 7.1.8 que es un programa de análisis cualitativo asistido por computadora el cual nos permitió asociar códigos o etiquetas con fragmentos de textos y videos. Así mismo, los códigos y patrones a fin de clasificarlos (Ruhl, 2004). En este programa creamos proyectos denominados Unidades Hermenéuticas que incluyen documentos primarios como citas, códigos, memos y finalmente la creación de redes. Se realizó un análisis temático, que es un método que permite identificar, organizar, analizar en detalle y proporcionar patrones o temas a partir de una cuidadosa lectura y relectura de la información recogida y así inferir resultados que propicien la adecuada comprensión/interpretación del fenómeno en estudio ${ }^{14}$.

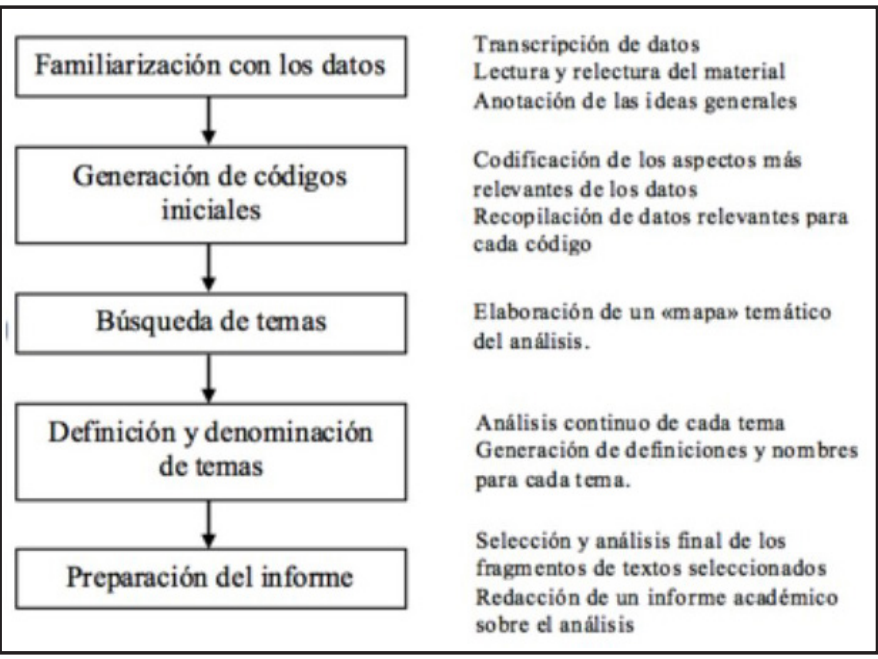

Figura 1. Fases del proceso de análisis de datos en el análisis temático.
Tomado de Braun V, Clarke V "Using thematic analysis in psychology", Qualitative Research in Psychology, 3(2), pág. 87. ${ }^{14}$

Para la realización del siguiente articulo fueron tomadas las consideraciones éticas como la declaración de Helsinski, el informe de Belmont, la declaración de los derechos humanos. Siendo respetadas las voluntades de los participantes quienes firmaron el consentimiento informado donde se le informaba que sus datos no serían revelados públicamente.

\section{RESULTADOS}

La información obtenida a través de entrevistas $y$ videos fue introducida en el programa Atlas.ti, donde se creó una unidad hermenéutica, a través de ella se generó citas, códigos y se creó las redes que nos dio los resultados que se expresan a continuación.

Las asignaturas cursadas durante la formación profesional en Estomatología son enfocadas a la atención del paciente adulto, y es común que la asignatura de odontopediatría se curse en los últimos ciclos de la vida pre profesional. Por lo tanto, el estudiante tiene dificultades para realizar un adecuado control de la conducta del niño al inicio de sus prácticas pre-profesionales. En una primera entrevista todos los alumnos coinciden en lo difícil que es atender a un niño la frase más repetida fue "... dificil atender a un niño ...", en esta frase que forma parte de la primera entrevista , se observa que el estudiante tiene cierta dificultad para realizar el control de la conducta del niño, los ánimos no son los mejores “... yo creo que no voy a pasar el curso doctora, no me creo capaz de atender un niño, estoy desanimado, mi niño hoy no se ha dejado hacer nada ..." lo dice Frank un estudiante que llevo su paciente pero no consiguió realizar ningún tratamiento, a pesar de haber aplicado todas las técnicas que conoció en la teoría, su error tal vez fue que la madre estuvo presente durante toda la atención. En una segunda visita pidió a la madre que no vaya a la consulta el niño llego con su tía quien se quedó en la sala de espera, luego Frank llega ".... doctora conseguí atender mi paciente...". Muchos de los estudiantes lograron el éxito una vez que aplicaron el control de la conducta del niño adecuado, solo 4 estudiantes decidieron cambiar de pacien- 
te no logrando el éxito. Ana una estudiante que atendió a una niña de 5 años manifestó "...... no he logrado atender a mi pacientita porque miro a su lado a un niño llorando y ella también se puso a llorar, si no hubiese pasado eso, quizás la hubiera convencido......", la llevo una vez más pero no consiguió atenderla apenas la niña se sentó comenzó a llorar, a pesar de haber aplicado todas las técnicas de control de la conducta del niño, en la tercera visita la niña llego llorando obligada por su madre, por lo que se decidió no atenderla "... no se puede atender a todos los niño en una misma clínica..., tiene que ser individual..." lo dice Ana, ya que generalmente la atención de los pacientes no son individualizadas.

En la segunda entrevista, los estudiantes mostraban confianza al hablar con los niños, la mayoría de los estudiantes estaban de acuerdo en usar la técnica decir - mostrar - hacer y la técnica de distracción audiovisual, llegaron a la conclusión que fue mucho más fácil atender a un niño utilizando ambas técnicas. "....yo primero le hablo al paciente le enseño las fotos que tome de sus dientes, le digo lo que voy a hacer y luego le muestro con un espejo, el niño luego me pide los lentes de realidad virtual, se los pongo y se deja atender...todos hacemos así y nos funciona..." lo dice Jesús.

En la tercera entrevista “...he aprendido cosas nuevas en el control de la conducta del niño, debo tener paciencia, ser dinámica con el niño para conseguir atenderlo ..." son las palabras de Judith

\section{CONOCIMIENTOS TEÓRICOS Y PRÁCTICOS}

ACERCA DEL CONTROL DE LA CONDUCTA DEL NIÑO

Las asignaturas cursadas durante la formación profesional en Estomatología son enfocadas a la atención del paciente adulto, y es común que la asignatura de odontopediatría se curse en los últimos ciclos de la vida pre profesional. Por lo que se hace común que el estudiante tenga dificultades para realizar un adecuado control de la conducta del niño. A pesar de encontrarse atendiendo un niño, inconscientemente tratan a un niño como si fuese un adulto y esto se ve reflejado cuando ellos responden "la conducta de un niño es diferente a un adulto" esto demuestra que esperaban que el comportamiento del niño sería similar o parecido al de un adulto. Así mismo, la respuesta "no se sabe la reacción del niño" invita a una reflexión donde se puede deducir que el estudiante ve ello como una dificultad para la atención odontológica expresándolo como "difícil atender a un niño" y es así, cómo ve la necesidad de que para la atención de un niño se necesita de paciencia que se ve reflejada en su respuesta. Todos los aspectos mencionados anteriormente influyen al momento del aprendizaje de los conocimientos teóricos y prácticos puesto que los estudiantes vienen con ideas preconcebidas acerca de la atención del niño manifestando la dificultad que supone atender a un niño aun conociendo las teorías para el control de la conducta.

\section{Aplicación de las técnicas del control DE LA CONDUCTA DEL NIÑO EN LA CLÍNICA DENTAL}

En la primera entrevista el estudiante manifiesta lo complicado que es aplicar las técnicas de control de la conducta a un niño "complicado aplicar la técnica al niño" coincidiendo que es necesario conocer las técnicas de control de la conducta. Considerando que para aplicar las técnicas ellos manifiestan que se debe tener confianza en la técnica a utilizar, va a depender de la conducta del niño y prueban diferentes técnicas para el control de la conducta. Con las diferentes técnicas utilizadas los estudiantes consideran que existen factores que influyen en la atención del niño y al control de la conducta. Los factores negativos para los estudiantes son la presencia de los padres en la atención a los niños, ya que con ellos el comportamiento es distinto que cuando el niño se encuentra solo, eso lo pudieron percibir en la segunda entrevista cuando no permitieron la entrada a los padres o tutores dentro la clínica dental, y se les pidió que observen por la puerta de cristal o las cámaras instaladas en la clínica dental. Otro factor fue la experiencia negativa previa, esto cuando el niño había sido tratado anteriormente por un dentista y tuvo una mala experiencia, y al momento de ser atendido rechazaba el tratamiento dental que se le realizaría, el estudiante manifiesta que al aplicar diferentes técnicas no obtenía los resultados esperados, hasta que después de tres citas gano la confianza del niño y solo ahí consiguió realizar un tratamiento dental. Si bien es cierto una clínica dental para estudiantes de estomatología no se encuentra divida por box por lo que los tratamientos realizados son fácilmente observados por el niño que se encuentra al lado, y cualquier comportamiento puede llegar a ser imi- 
tado, por ejemplo, si un niño llegase a llorar el niño que se encuentre próximo a este puede cambiar su comportamiento y comenzar también a llorar. Por lo que se tomó la decisión que los niños con mal comportamiento se retiren de la clínica hasta el cambio de comportamiento, otra opción sería contar con espacios individualizados para cada niño de tal forma que no dé pie a la imitación un mal comportamiento. Después de tomada la decisión se evidencio que ya no existía el problema de la imitación de un mal comportamiento. El temor al daño por parte del niño también fue controlado mediante la desensibilización del niño, es decir, explicando al niño cada uno de los procedimientos antes de ser realizados.

\section{DISCUSIÓN}

Existe pocos estudios cualitativos relacionado con el presente tema, las universidades a nivel mundial se encuentran dedicadas básicamente a la atención integral del paciente adulto, son escasas aquellas que se dedican a la atención integral del paciente pediátrico quedando la enseñanza restringida a una especialidad. Ello se ve reflejado en la malla curricular de las diferentes universidades, donde se interpreta que existen pocas asignaturas destinadas a dar un soporte básico a la asignatura principal como lo es la odontopediatría ya que el tema de psicología del niño se trata someramente. Siendo el tema mencionado importante en la formación académica del estudiante de odontología. Existen estudios cualitativos como el de Valare$\mathrm{zo}^{15}$ que se han encargado de analizar la malla curricular de escuelas profesionales como medicina y enfermería en el Perú encontrando un bajo nivel, los estudios realizados en analizar mallas curriculares en odontología aún no han sido analizados o por lo menos no han sido publicados. Las mallas curriculares planteadas en la mayoría de instituciones educativas concernientes al área de odontopediatría se ve reflejada en la falta de confianza del estudiante al momento de la atención del paciente pediatrico ${ }^{16}$.

Por otro lado, algunos estudios realizados en profesiones a fines indican que la confianza del estudiante depende de la satisfacción y estimulo de sus docentes y esto se ve reflejado en el presente estudio, cuando el docente constantemente inspira confianza al alumno para continuar con el tratamiento del niño. Por lo que, es necesario esta- blecer que la enseñanza es un ítem fundamental en este investigación nos deja claro que sin ella no puede ser posible desarrollar la confianza ni el estímulo en el estudiante ${ }^{18}$.

\section{CONCLUSIONES}

Se concluye que el estudiante no adquiere las habilidades suficientes para lograr el control de la conducta del niño al momento de su atención odontológica, a pesar de tener los conocimientos teóricos. Pero ello se va supliendo siempre y cuando el estudiante toma conciencia y pone en práctica lo aprendido en la teoría. El estudiante debe tomar conciencia de la importancia de conocer y practicar las técnicas del control de la conducta, de lo contrario ellos se pueden ver influenciados al uso de productos farmacológicos en niños que a corto o largo plazo pueden ser dañinos para la salud.

\section{BIBLIOGRAFIA}

1. Marshall C, Rossman GB. Designing Qualitative Research. SAGE Publications; 1999. 244 p.

2. Seidman I. Interviewing as Qualitative Research: A Guide for Researchers in Education and the Social Sciences. Edición: 3rd Revised edition. New York: Teachers' College Press; 2006. 176 p.

3. Do C. Applying social learning theory to children with dental anxiety. J Contemp Dent Pract. 15 de febrero de 2004;5(1):126-35.

4. Angelo Z, Polyvios C. Alternative practices of achieving anaesthesia for dental procedures: a review. J Dent Anesth Pain Med. abril de 2018;18(2):79-88.

5. Jamali Z, Najafpour E, Ebrahim Adhami Z, Sighari Deljavan A, Aminabadi NA, Shirazi S. Does the length of dental procedure influence children's behavior during and after treatment? A systematic review and critical appraisal. J Dent Res Dent Clin Dent Prospects. 2018;12(1):68-76.

6. Asl AN, Shokravi M, Jamali Z, Shirazi S. Barriers and Drawbacks of the Assessment of Dental Fear, Dental Anxiety and Dental Pho- 
bia in Children: A Critical Literature Review. J Clin Pediatr Dent. 2017;41(6):399-423.

7. Aminabadi NA, Oskouei SG, Farahani RMZ. Dental treatment duration as an indicator of the behavior of 3-to 9-year-old pediatric patients in clinical dental settings. J Contemp Dent Pract. 1 de septiembre de 2009;10(5):E025032.

8. Tsoi AK, Wilson S, Thikkurissy S. A Study of the Relationship of Parenting Styles, Child Temperament, and Operatory Behavior in Healthy Children. J Clin Pediatr Dent. 2018;42(4):273-8.

9. Wilson S, Cody WE. An analysis of behavior management papers published in the pediatric dental literature. Pediatr Dent. agosto de 2005;27(4):331-8.

10. Flanagan Borquez A. Experiencias de estudiantes de primera generación en universidades chilenas: realidades y desafíos. Rev Educ Super. 1 de julio de 2017;46(183):87-104.

11. Guzmán Gómez C, Ramos S, Lucy C. Experiencias, vivencias y sentidos en torno a la escuela y a los estudios: Abordajes desde las perspectivas de alumnos y estudiantes. Rev Mex Investig Educ. diciembre de 2015;20(67):1019-54.

12. Moustakas C. Phenomenological Research Methods. SAGE; 1994. 210 p.

13. Balcázar Nava P, López-Fuentes G-A, Ivonne N, Peña G, Margarita G, Moysén Chimal A. Investigación cualitativa [Internet]. Universidad Autónoma del Estado de México; 2013 [citado 4 de junio de 2018]. Disponible en: http://repositorio.minedu.gob.pe/handle/123456789/4641

14. Braun V, Clarke V. Using thematic analysis in psychology. Qual Res Psychol. 1 de enero de 2006;3(2):77-101.

15. Valarezo-García C, Solis Cartas U, Valarezo Espinosa P. Integración de la medicina alternativa en la malla curricular de las carreras de Medicina y Enfermería de las universidades peruanas. Educ Médica [Internet]. 24 de abril de 2018 [citado 7 de junio de 2018]; Disponible en: http://www.sciencedirect.com/science/article/pii/S1575181318300858

16. Castro Rodríguez Y, Sihuay-Torres K, Perez-Jiménez V. Producción científica y percepción de la investigación por estudiantes de odontología. Educ Médica. 1 de enero de 2018;19(1):19-22.

17. Uriarte S, Ponce G, Bernal M. Vivencias cotidianas en espacios clínicos del estudiante de enfermería. Enferm Univ. 1 de julio de 2016;13(3):171-7.

18. Rodríguez-Díez MC, Díez N, Merino I, Velis JM, Tienza A, Robles-García JE. La simulación mejora la confianza de los estudiantes para adquirir competencias en urología. Actas Urol Esp. 2016;367-72.

\section{AUTOR DE CORRESPONDENCIA:}

Marisel Roxana Valenzuela Ramos

Correo electrónico:

mvalenzuela@crece.uss.edu.pe Universidad

Señor de Sipán

Teléfono móvil: 00511956291247 